\title{
Random pulse input versus continuous current plus white noise: Are they equivalent?
}

\author{
Stuart Feerick*, Jianfeng Feng, David Brown \\ Laboratory of Computational Neuroscience, The Babraham Institute, Cambridge CB2 4AT, UK
}

Accepted 13 January 2000

\begin{abstract}
We compared the behavior of the Hodgkin-Huxley $(\mathrm{HH})$ model subjected to two input regimes: firstly, discrete pulses from a Poisson process, and then to a diffusion approximation, constructed to share statistical properties with the discrete pulse input. The results show that under a wide range of physiologically plausible input conditions the diffusion process is equivalent to the pulse process, in terms of output interspike interval distribution. In this respect, the $\mathrm{HH}$ model differs from the integrate and fire model, which has been widely used to address a variety of questions in computational neuroscience. (C) 2000 Published by Elsevier Science B.V. All rights reserved.
\end{abstract}

Keywords: Hodgkin-Huxley model; Stochastic synaptic input; The usual approximation

\section{Introduction}

For a wide range of neuronal models it is common to model the input as a Poisson process, or more generally a renewal process, which is appropriate for a neuron subjected to a stream of post-synaptic potentials, characterized by a short rise time which may be considered as a step process at the axon hillock. The effect of such input has in the past been analyzed for a very simple neuronal model, the integrate and fire model (IF), both with and without leakage, and with and without reversal potentials $[5,1,2]$. However, the resulting system of differential difference equations has no useful

\footnotetext{
* Corresponding author. Tel.: + 44-1223-496256; fax: + 44-1223-496031.

E-mail address: smf26@cam.ac.uk (S. Feerick).
} 
closed-form solution except for some special cases. Moreover, for the biologically more interesting biophysical models, such as the Hodgkin-Huxley $(\mathrm{HH})$, an analytical solution is impossible.

One way to simplify, and thus better understand, these problems has been to replace the discrete pulses with a process composed of a constant current plus small randomly sized steps at small intervals of time, which shares statistical properties with the original Poisson process, i.e. a diffusion process. The simplest method to obtain this approximation is to arrange for the diffusion process to share the same first and second infinitesimal moments with the pulse process, known as the usual approximation. This approximation is realised in the limit as the rate of the Poisson process tends to infinity and the size of the inputs to zero. We further investigated the distribution of the output ISI, and inspection of the ISI histograms revealed the distributions to be extremely similar.

In summary, the HH model exhibits physiologically more plausible behavior when stimulated with random input, due to its non-linearity and memory, which smooth out input discontinuities. This allows the diffusion approximation to work well in a wide region of parameter space. We are extending this work to other physiological models and correlated inputs.

\section{Models and methods}

We use the following $\mathrm{HH}$ model:

$$
C \frac{\mathrm{d} V}{\mathrm{~d} t}=I_{\mathrm{syn}}+g_{K} n^{4}\left(V-V_{K}\right)+g_{N a} m^{3} h\left(V-V_{N a}\right)+g_{L}\left(V-V_{L}\right),
$$

where

$$
\frac{\mathrm{d} n}{\mathrm{~d} t}=\frac{n_{\infty}-n}{\tau_{n}}, \quad \frac{\mathrm{d} m}{\mathrm{~d} t}=\frac{m_{\infty}-m}{\tau_{m}}, \quad \frac{\mathrm{d} h}{\mathrm{~d} t}=\frac{h_{\infty}-h}{\tau_{h}}
$$

and

$$
\begin{array}{ll}
n_{\infty}=\frac{\alpha_{n}}{\alpha_{n}+\beta_{n}}, & m_{\infty}=\frac{\alpha_{m}}{\alpha_{m}+\beta_{m}}, \quad h_{\infty}=\frac{\alpha_{h}}{\alpha_{h}+\beta_{h}} \\
\tau_{n}=\frac{1}{\alpha_{n}+\beta_{n}}, & \tau_{m}=\frac{1}{\alpha_{m}+\beta_{m}},
\end{array}
$$

Here $I_{\text {syn }}$ is the synaptic current applied to the model. The model parameters are as in Ref. [4]. We apply two input regimes:

- Inputs are modelled in terms of excitatory and inhibitory synaptic inputs into a neuron. Inputs are independent, time homogeneous Poisson processes, and we have $N_{\mathrm{e}}$ excitatory inputs, each of magnitude $a_{\mathrm{e}}$ and mean rate $\lambda_{\mathrm{e}}$, and $N_{\mathrm{i}}$ inhibitory inputs, each of magnitude $a_{\mathrm{i}}$ and mean rate $\lambda_{\mathrm{i}}$; 
- We compare this input to a diffusion process sharing the same mean and variance as the Poisson input [3], $I_{\text {syn }}=\mu+\sigma B(t)$, where $B(t)=\mathrm{d} \xi(t) / \mathrm{d} t$ with $\xi(t)$ as the standard Brownian motion and $\mu$ and $\sigma$ are given by $\mu=a_{\mathrm{e}} N_{\mathrm{e}} \lambda_{\mathrm{e}}-a_{\mathrm{i}} N_{\mathrm{i}} \lambda_{\mathrm{i}}$, and $\sigma^{2}=a_{\mathrm{e}}^{2} N_{\mathrm{e}} \lambda_{\mathrm{e}}+a_{\mathrm{i}}^{2} N_{\mathrm{i}} \lambda_{\mathrm{i}}$.

Simulations were performed over a wide variety of physiologically plausible input regimes, and in each case 2000 output interspike intervals were obtained.

\section{Results}

We begin our simulation using small inputs $\left(a_{\mathrm{e}}=a_{\mathrm{i}}=0.5 \mathrm{mV}\right)$. The threshold for firing in this model is at around $-58 \mathrm{mV}$ compared to the resting potential of $-65 \mathrm{mV}$, and so around 20 input spikes are required to cause spiking. Results (Fig. 1) show a close agreement between Poisson input and the diffusion approximation. Analysis of these results shows no significant difference between them. Repeating the experiment with larger inputs $\left(a_{\mathrm{e}}=a_{\mathrm{i}}=2.0 \mathrm{mV}\right)$ show a similar close agreement (Fig. 2), though in this case there are significant differences for low $N_{\mathrm{e}}$ and $N_{\mathrm{i}}$. Given the theoretical underpinning of the diffusion approximation, this is perhaps unsurprising. For both of the above inputs, the coefficient of variation of the output interspike interval was also recorded, and shows similar close agreement between the two input regimes. Further investigation using ISI histograms reveals that the similarity expressed by the first- and second-order statistics is repeated in the distribution (see, for example, Fig. 3). Here we are unable to detect any significant difference between the input regimes.

\section{Discussion}

Our numerical simulations show close agreement for mean interspike interval (ISI) between the Poisson input case and its associated diffusion approximation over the

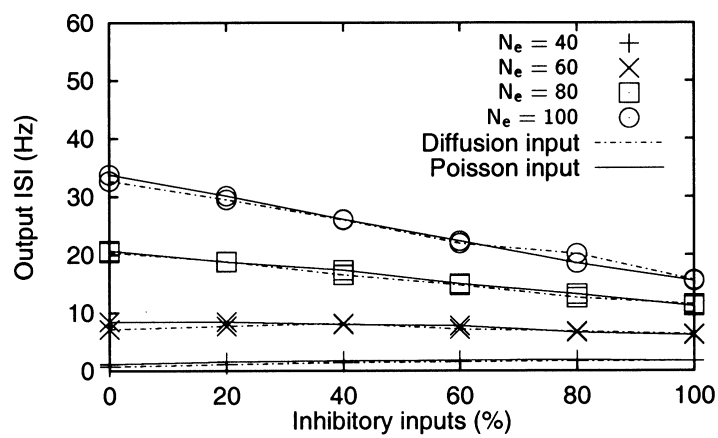

Fig. 1. Output frequency $(\mathrm{Hz})$ of a Hodgkin-Huxley model neuron receiving $N_{\mathrm{e}}$ excitatory inputs of magnitude $a_{\mathrm{e}}=0.5 \mathrm{mV}$ and $N_{\mathrm{i}}=r * N_{\mathrm{e}}$ inhibitory inputs of magnitude $a_{\mathrm{i}}=0.5 \mathrm{mV}$. All inputs have mean frequency $100 \mathrm{~Hz}$. 


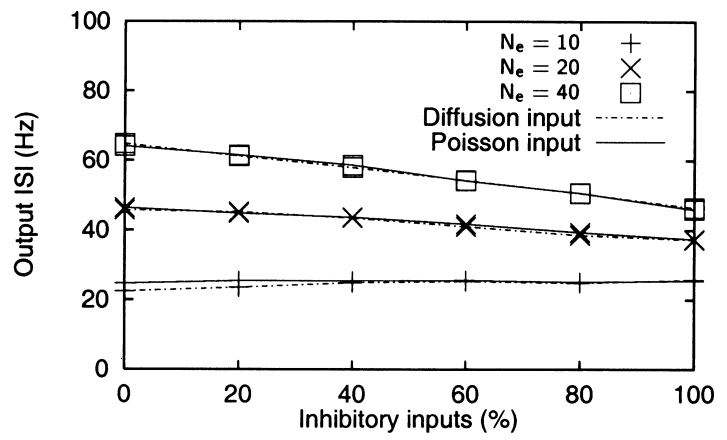

Fig. 2. Output frequency $(\mathrm{Hz})$ of a Hodgkin-Huxley model neuron receiving $N_{\mathrm{e}}$ excitatory inputs of magnitude $a_{\mathrm{e}}=2.0 \mathrm{mV}$ and $N_{\mathrm{i}}=r * N_{\mathrm{e}}$ inhibitory inputs of magnitude $a_{\mathrm{i}}=2.0 \mathrm{mV}$. All inputs have mean frequency $100 \mathrm{~Hz}$.

\section{Poisson input}
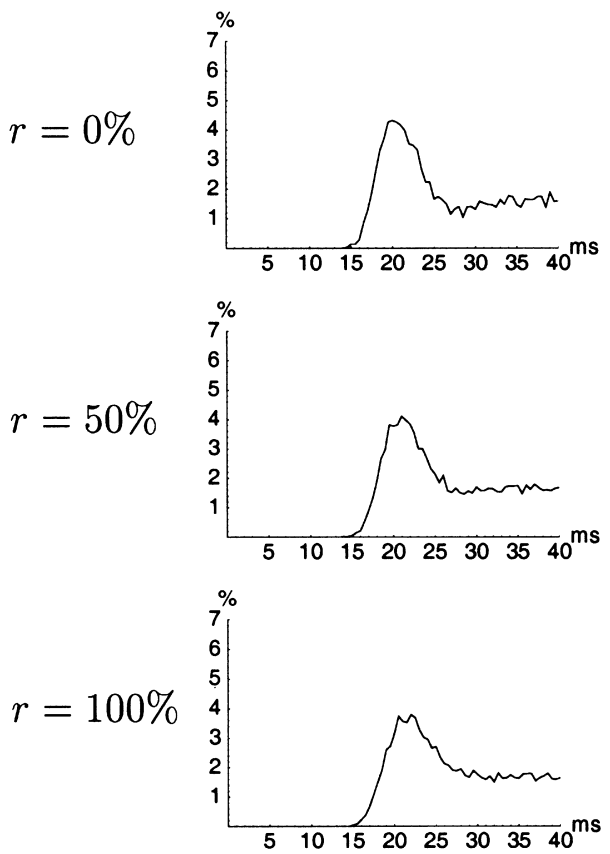

Diffusion input
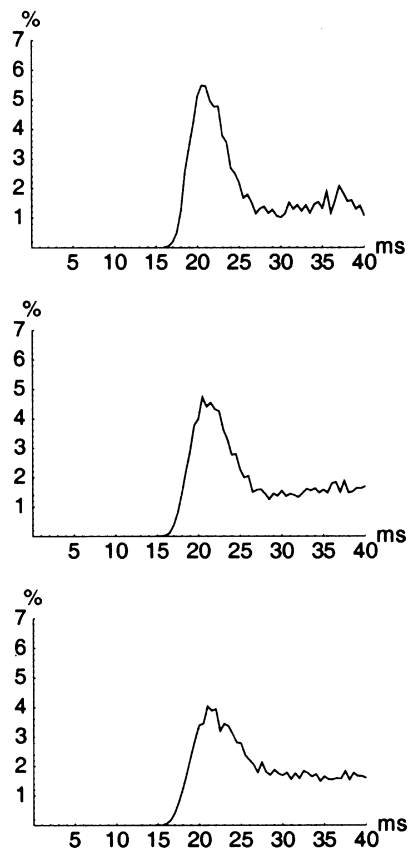

Fig. 3. Histograms showing percentage of interspike intervals of a Hodgkin-Huxley model neuron receiving $N_{\mathrm{e}}=5$ excitatory inputs of magnitude $a_{\mathrm{e}}=2.0 \mathrm{mV}$ and $N_{\mathrm{i}}=r * N_{\mathrm{e}}$ inhibitory inputs of magnitude $a_{i}=2.0 \mathrm{mV}$. All inputs have mean frequency $100 \mathrm{~Hz}$.

whole range of parameter space. Corresponding results were typically less than two standard errors (of the estimation error in the simulation experiments) apart. There was no evidence of systematic difference between the two sets of results. Similarly, coefficient of variation $(\mathrm{CV})$ of the output ISI was estimated well by the diffusion 
approximation, the two estimates being well within one standard error of each other. It should be noted, in particular, that the diffusion approximation works well even when the input is of low intensity or high magnitude; cases when the assumptions underlying the approximation break down.

In previous work with the IF model [6], the diffusion approximation was shown to have limitations: in particular, the output frequency under the diffusion approximation was shown to be systematically higher, by around $10 \%$, than that obtained with discontinuous inputs. For the HH model, we observe no such discrepancies, as we state above. The case of exact balance has attracted considerable attention, both for its physiological significance and the behavior of the IF model, which has very high mean ISI, and a high CV [1]. Due to these features, the diffusion approximation breaks down for the IF model. The HH model, however, exhibits no such behavior, and both ISI and CV remain relatively constant even when excitatory and inhibitory inputs are balanced; and again the diffusion approximation holds. This is further evidence of the more robust nature of the HH model compared to the IF model.

\section{Acknowledgements}

This work was financially supported by the BBSRC and a grant from the Royal Society.

\section{References}

[1] D. Brown, J. Feng, Is there a problem matching real and model CV(ISI)? Neurocomputing 26 (7) (1999) 87-91.

[2] J. Feng, D. Brown, Coefficient of variation of interspike intervals greater than 0.5. How and when? Biol. Cybernet. 80 (1999) 291-297.

[3] F.B. Hanson, H.C. Tuckwell, Diffusion approximation for neuronal activity including synaptic reversal potentials. J. Theoret. Neurobiol. 2 (1983) 127-153.

[4] A.L. Hodgkin, A.F. Huxley, A quantitative description of membrane current and its application to conduction and excitation in nerve. J. Physiol. 117 (1952) 500-544.

[5] P. Lánsky, M. Musila, Variable initial depolarisation in Stein's neuronal model with synaptic reversal potentials. Biol. Cybernet. 94 (1991) 285-291.

[6] M. Musila, P. Lánsky, On the interspike intervals calculated from diffusion approximations of Stein's neuronal model with reversal potentials. J. Theoret. Biol. 171 (1994) 225-232.

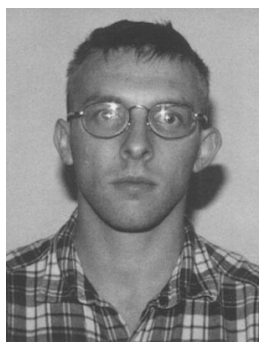

Stuart Feerick received a B.A. in mathematics from Cambridge University in 1993, and is currently pursuing a doctorate in mathematical biology at the Babraham Institute, also in Cambridge. 


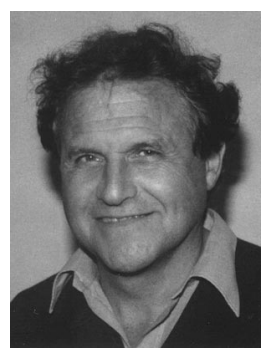

David Brown is Head of the Laboratory of Computational Neuroscience at the Babraham Institute, Cambridge, UK. His research interests are in stochastic modelling and analysis of neuronal systems (particularly neuroendocrine systems and the olfactory bulb), and statistical analysis of electrophysiological data.

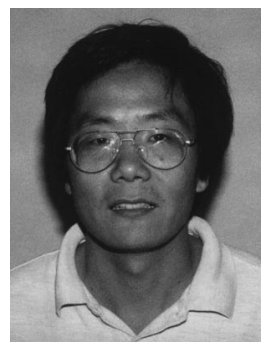

Dr. J. Feng is a senior research scientist (project leader) at the Babraham Institute, Cambridge CB2 4AT, UK. He is mainly interested in modelling neuronal systems, from single-to-system levels. 